\title{
Tap and Push: Assessing the Value of Direct Physical Control in Human-Robot Collaborative Tasks
}

\author{
Brian Gleeson, Katelyn Currie, Karon MacLean, Elizabeth Croft \\ University of British Columbia
}

\begin{abstract}
In this paper, we compare a touch-based human-to-robot command scheme with traditional button commands in a series of human-robot collaborative assembly tasks. We find a mapping between command style and task outcome that depends on task complexity and is influenced by robot "feel." In our direct touch-based scheme, the user commands the robot through direct physical contact by tapping and pushing the robot. With a small, compliant desktop robot and a simple, scripted, bolt insertion task, button commands performed slightly better than direct physical commands in quantitative task performance metrics and qualitative user preference. In a second study with a human-scale, stiffer robot arm, physical commands performed better than button commands in a more complex and less scripted bolt insertion task, which greatly outperformed using buttons in a cooperative positioning task. We conclude that commanding a robot through direct force-transmitting contact can decrease task completion time, aid in teamwork, and improve user experience in appropriately chosen tasks. We achieve our haptic commands using only robot position sensors, demonstrating that direct, intuitive physical command is an option for existing position-controlled industrial robots.
\end{abstract}

Keywords: Physical human-robot interaction, human-robot cooperative work, industrial assembly, direct physical interface, direct manipulation, haptic HRI

\section{Introduction}

Advances in robot control, sensing, and intelligence are rapidly expanding the potential for closeproximity human-robot collaborative work. In many contexts, a robot's potential strength, precision, and process knowledge can productively complement human perception, dexterity, and intelligence to produce a highly synergistic human-robot team. Such interaction, however, requires task-appropriate communication methods to facilitate human-robot teamwork.

We hypothesize that direct physical commands of a robot by a human partner may be easier, faster, and more intuitive than existing button-based command interfaces (Fig. 1) and may provide a command alternative in situations where existing methods are impractical or obstructive. As an interaction method, physical commands can be thought of as a form of direct manipulation. In human-computer interaction, direct manipulation is a highly flexible, intuitive means of interacting with software (e.g., Hutchins, Hollan, \& Norman, 1985), and we propose that direct manipulation, in the form of tapping, pushing, and grabbing robot limbs, may be similarly powerful in human-robot interaction (HRI). A good haptic command interface would minimize the cognitive demand for robot control and allow both novice and expert users to command a robot without the need for specialized training (Adams, 2002).

Authors retain copyright and grant the Journal of Human-Robot Interaction right of first publication with the work simultaneously licensed under a Creative Commons Attribution License that allows others to share the work with an acknowledgement of the work's authorship and initial publication in this journal.

Because of a conflict of interest with one author as a guest editor, this paper was handled solely by the co-editor, Antonio Frisoli. The decision to accept or reject this paper was made without consulting the authoring guest editor.

Journal of Human-Robot Interaction, Vol. 4, No. 1, 2015, Pages 95-113, DOI 10.5898/JHRI.4.1.Gleeson 
Furthermore, haptic command would be an ideal means of interaction when other means are impractical. For example, a user working collaboratively with a robot inside the chassis of a car is constrained in command options: chassis occlusion impedes visual detection of gestures, a high environmental noise level interferes with spoken commands, and a button interface may be impractical due to a need to use both hands on work piece or robot; furthermore, it can be difficult to specify a desired behavior using this abstract channel (Adams, 2002). We posit that it would be useful if the user could command the robot by exerting forces at any point along the robot or even on the work piece held by the robot.

In this paper, we primarily consider industrially relevant tasks. While industrial robots are a key factor in advanced flexible manufacturing, current programming and interaction methods centered around teach pendants hinder the effective adoption of robots in many tasks (Pieska, Kaarela, \& Saukko, 2012). In addition to industrial uses, direct physical command may also have applications in other areas, such as home healthcare robotics, where a highly intuitive interface is important, or therapy robotics, where physical contact with the robot may be socially beneficial.

The technical feasibility of this approach is supported by prior demonstration of several ways to sense contact between a human and robot, a s summarized in Section 2.3, but there has been minimal exploration of how to exploit this for collaborative purposes. Our present goal is to evaluate whether a straightforward implementation of a direct physical command scheme is useful in meditating human-robot collaboration and to compare its utility in various contexts with more traditional means of robot command.

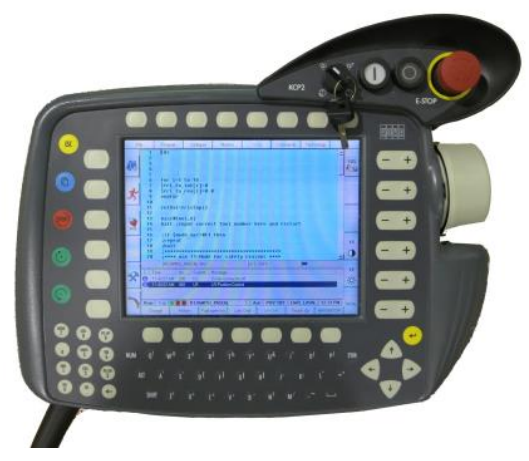

Figure 1. An example teach pendant (Kuka LWR controller). Such interfaces are illsuited for human-robot collaborative work.

\subsection{Approach \& Contributions}

In this paper, we present two experiments comparing direct physical and traditional button commands in human-robot cooperative tasks and discuss insights as to the relative value and appropriateness of these two approaches to real-time human control of a robot collaborator's behavior. In our first experiment, featuring a simple collaborative assembly task, we ask, "Is direct physical command feasible, and how do users regard it relative to traditional button command?" We found that users could use direct touch for command, but it did not improve task performance, and that excessive robot compliance degraded the interaction. In a second experiment, conducted on a stiffer robot, we asked the more nuanced question, "How do the relative performance and acceptability of physical and traditional button commands shift in more complex tasks?" We found that physical commands were well suited to more complex and less scripted tasks, improving task performance in both qualitative and quantitative metrics. We make the following contributions: 
- Objective evidence that direct physical commands can be a powerful tool for human control of a robot in collaborative tasks

- Insight into the types of human-robot tasks most suited to direct physical commands

- A demonstration that direct-touch command can be achieved using only the control and sensor abilities of common industrial robots

What follows is an overview of haptic or physical commands in HRI and its enabling technology. We summarize our methods for estimating contact force and our scheme for direct physical commands and then describe our experiment tasks. Finally, we present, discuss, and draw conclusions from our results.

\section{Background}

\subsection{HRI in Human-Robot Collaborative Work}

Industrial processes and products are now changing on a short time scale, driving a need for flexible automation with robots that can be quickly and easily commanded in changing assembly tasks (Brogårdh, 2007). The defacto standard interface in industry is a teach pendant featuring an array of buttons, or in recent developments, a touch screen GUI (Azin, Balazs, Trygve, \& Gabor, 2012). While button and teach pendant interfaces have developed significantly, they are insufficient for the types of rapidly changing tasks faced by modern industry (Pieska et al., 2012).

Potential modes for explicit human-robot communication include speech, gesture, and haptic communication, while implicit communication channels include manipulative gestures, proactive task execution, and physiological signals (Bauer, Wollherr, \& Buss, 2008). Examples of studies on communication for human-robot collaboration include work on gesture (Gleeson, MacLean, Haddadi, Croft, \& Alcazar, 2013), speech (Chao \& Thomaz, 2012), and augmented reality (Green, Billinghurst, Chen, \& Chase, 2008). Other tools for more fluid collaborative work include a system to adapt a robot's actions based on observations of its partner's actions (Nikolaidis et al., 2013) and a system for controlling a robot using task-level commands, as opposed to more tradition step-by-step commands (Shah, Wiken, Williams, \& Breazeal 2011). These methods enable intuitive, flexible communication between human and robot, expanding the capabilities of human-robot teams.

Herein, we focus on haptic (direct physical) human-to-robot communication for collaborative work as a supplemental method or for use in tasks where other communication modes are impractical. We hypothesize that direct manipulation through haptic commands can facilitate fluent, efficient human-robot collaboration.

\subsection{Haptic and Physical Robot Command}

Several groups have demonstrated advances in human-robot contact detection and haptic sensing, but few have studied the interactions that touch commands could facilitate. Most commonly, contact detection is used as a safety mechanism where the robot stops or retreats in response to contact (e.g., Haddadin, Albu-Schaffer, De Luca, \& Hirzinger, 2008; Suita et al., 1995), or to switch command modes, allowing the user to push and pull the through the workspace (e.g., Erden \& Tomiyama, 2010; Frigola, Casals, \& Amat, 2006). In Haddadin et al. (2008), different controlmode switching behaviors were formally compared, showing that users felt safest when collisions resulted in a switch to a passive gravity compensation mode.

Two direct-touch applications are collaborative object manipulation and learning by demonstration. Wojtara et al. (2009) compared several control methods for collaborative positioning of large objects using direct physical interaction, and Lee (2010) developed a prototype robot for collaborative manipulation in construction tasks. 
The related task of collaborative object carrying has been studied by Parker \& Croft (2012), among others, and object handover, a type of collaborative manipulation, has been investigated by Strabala et al. (2013), Chan, Parker, Van der Loos, and Croft (2013), and others. In learningby-demonstration tasks, direct physical manipulation allows users to easily specify robot trajectories, even in constrained workspaces (e.g., Wrede et al., 2013).

Others have used direct physical touch for robot guidance, for example, of a mobile robot (Frémy, Ferland, Lauria, \& Michaud, 2014). In a study using an anthropomorphic nurse robot, users could guide the robot and position the robot's arms by exerting forces on the hand (Chen \& Kemp, 2010). In this experiment, conceptually similar to our study, direct guidance was compared to a game controller interface in a patient lifting task and was found to improve task performance and robot usability. These studies show a benefit to direct physical guidance with a human in a supervisory role; we expand on this foundation by studying the use of direct physical guidance along with higher-level task commands, in a range of tasks with semiautonomous robot motion, human-robot turn-taking, and with the human as both a supervisor and collaborator.

\subsection{Human-Robot Contact Detection}

For any touch-based human-robot interaction, one must first detect contact between the human and robot - depending on the task; this may also entail force magnitude, direction, and exact location of the touch. For our experiments, we developed a method of contact detection that is tailored to our intended applications, but other equally effective means of contact detection have been proposed by others. These ideas include direct measurement through force sensors (Voyles \& Khosla, 1995), joint torque sensors (Bischoff et al., 2010), various types of "robot skin" (Mukai, Onishi, Odashima, \& Hirano, 2008), or sensorless methods (de Luca, Albu-Schaffer, Haddadin, \& Hirzinger, 2006). The technology to support meaningful haptic interaction between humans and robots is available; the challenge now is to develop and evaluate effective methods of haptic interaction and control.

\section{Methods}

We conducted two experiments evaluating direct physical commands in human-robot collaborative tasks. The first was exploratory to validate the general concept and highlight needed interaction refinements: it was conducted on a desktop Phantom Omni and featured a simple, scripted bolt insertion task. The second experiment examined the interaction between human-torobot control scheme and task parameters, based on refined physical interaction. A user directed a human-scale Barrett WAM $^{\mathrm{TM}}$ robot arm in two tasks: a more complex bolt insertion task and a cooperative positioning task. All tasks represented important industrial assembly functions, drawn from a previous analysis of real assembly line operations (Gleeson et al., 2013).

\subsection{Contact Detection}

The technical enabler for physical command implementation is force contact detection, which has been already accomplished in a variety of ways (Section 2.3). We chose to use a sensorless method to demonstrate that physical robot command is a practical option for end users of industrial robots in real-world applications. We implemented a method functionally similar to de Luca et al. (2006), although our method operated under constraints proposed by Geravand, Flacco, and de Luca (2013): we assume that the robot operates under position control, the user does not have access to the low-level controllers, and the user may not have a full dynamic model of the robot. We estimate the magnitude and direction of contact forces at any point from controller effort and use this estimation to drive our position controllers. When available, force sensors or other methods of direct contract detection would only make the implementation of haptic commands easier and improve the fidelity of the interaction. 


\subsection{Scheme for Direct Physical Robot Command}

Our physical command system is based upon two basic types of interaction: taps and pushes. Qualitatively, we define a tap as a brief contact, where the user taps the robot with her hand, exerting an impulse force on the robot but not creating any significant movement in the robot. A push is a sustained contact event, where the user applies a force (a push or a pull) to displace the robot. We measured the direction of tap and push inputs, with different directions mapped to different commands, as described under the Experiment Design section. In addition to taps and pushes, the robot detected other contacts and collisions, responding with a force-limiting behavior to ensure the comfort of the user.

The parameters for the interactions are as follows: minimum tap force $=1.5 \mathrm{~N}$, minimum push displacement $=20 \mathrm{~mm}$. In collisions with the user, the maximum collision force was restricted to $0.8 \mathrm{~N}$ (assuming collision with a stationary hand or arm).

Although our robots were always position-controlled and lacked force sensing, pushing a robot resulted in an experience similar to interacting with a robot under impedance control. We achieved this by updating the commanded position of the robot in response to estimated force inputs. While this is not true impedance control, we use the term in this paper for simplicity.

\subsection{Robots and Controllers}

Phantom Omni. Our first experiment employed a 3-DoF Sensible Phantom Omni robot (currently marketed as the Geomagic Touch; Fig. 2) While the Omni is designed to be used as a haptic interface, it can also be position-controlled and used as a small robot arm. The Omni has a maximum output force of $3.3 \mathrm{~N}$ and a workspace of approximately $16.3 \times 12.2 \times 7.1$ (width $\times$ height $\times$ depth) $\mathrm{cm}$. The Omni also features an un-actuated 3-DoF wrist and a stylus attachment for use in haptic interactions; in our experiments, we removed the stylus and immobilized the wrist joint with an elastic strap.

We chose the Omni for this first experiment primarily for safety, both real and perceived. The Omni is an inherently safe device, built for human contact. Equally important, subjects were likely to perceive it as safe, due to its small size and overall appearance as a consumer device.

We drove the Omni with a $1 \mathrm{kHz}$ PID position controller, using a PC running Windows 7 and the Sensable OpenHaptics Toolkit API. All robot motions followed human-like, minimumjerk quintic equation-planned trajectories, which have been shown to make people feel safer in human-robot collaborative tasks (e.g., Huber, Rickert, Knoll, Brandt, \& Glasauer, 2008).

Barrett WAM. We conducted our second experiment with a 4-DoF (wrist removed) Barrett WAM robot (Fig. 2), which is a high performance, human-scale, backdrivable robot arm. Compared to the Omni, the WAM arm has a larger workspace (reach $\sim 1 \mathrm{~m}$ ), is capable of exerting higher forces $(\max \sim 40 \mathrm{~N})$, and has high stiffness $\left(1.5^{*} 10^{6} \mathrm{~N} / \mathrm{m}\right)$. We operated the robot with the Barrett Haptic Ball end effector-a steel sphere of $50.8 \mathrm{~mm}$ diameter. We drove the WAM with a $1 \mathrm{kHz}$ PID position controller using a PC running Xenomai real-time Linux v.2.5.5.2 and using the btclient ${ }^{\mathrm{TM}}$ libraries for current sensing and motion control. As before, all robot trajectories were minimum-jerk quintic splines.

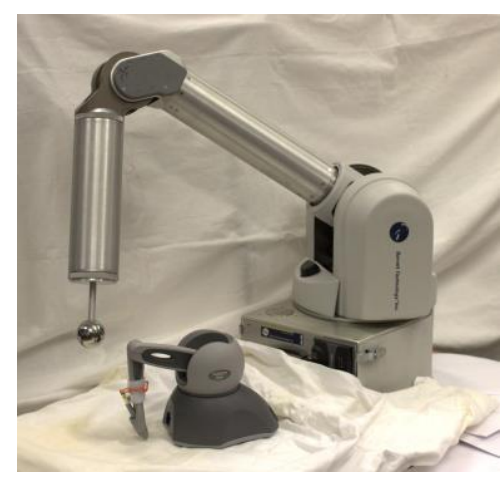

Figure 2. Robots used: Phantom Omni (front, Experiment 1) and Barrett WAM (back, Experiment 2). 
Gleeson et al. Haptic Control in H-R Collaborative Tasks

\section{Experiment Design and Human-Robot Tasks}

\subsection{Overview of Experimental Tasks}

We studied command method performance in three different human-robot collaborative tasks: Task A: Simple Bolt Insertion (Experiment 1), Task B: Complex Bolt Insertion (Experiment 2), and Task C: Cooperative Positioning (Experiment 2). Tasks were designed with differing levels of complexity (see Table 1; also see the supplementary video for demonstrations of the tasks).

Table 1. Key measures of complexity for the three experimental tasks.

\begin{tabular}{|c|c|c|c|}
\hline & Simple Bolt & Complex Bolt & Positioning \\
\hline Task Order & Scripted & Variable & Variable \\
\hline Branching* & 2 & 3 & 4 \\
\hline N buttons & 3 & 3 & 9 \\
\hline N commands** & 4 & 12 & $>50$ \\
\hline Command type & Discrete & Discrete & Discrete \& Continuous \\
\hline Collaboration type & Turn-taking & Turn-taking & Cooperative \\
\hline Experiment & Experiment 1 & Experiment 2 & Experiment 2 \\
\hline
\end{tabular}

*the number of possible directions the robot could move from each waypoint

**the minimum number of button commands required to complete the task

\subsubsection{Task A: Simple Bolt Insertion Task}

Fastener insertion is a common assembly task (Gleeson et al., 2013). While generally human-performed, fastener insertion is an example where a robot and human's capabilities could complement one another. The human, with better perception and dexterity, performs the delicate task of threading fasteners, while the robot, with greater strength (and, potentially, torque sensing), tightens the bolts. Variations on collaborative fastener insertion have been used before as a benchmark human-robot collaborative task (Nikolaidis et al., 2013). Our task was mostly scripted, but we introduced minor task variations to simulate a varied interaction, representing variability in parts, work pieces, assembly plans, or error correction.

A typical trial simulated placement and tightening of four bolts. Participants picked up bolts and washers from open boxes, placed a bolt with a washer in one of four locations on a board (corners of a $150 \times 160 \mathrm{~mm}$ rectangle-Fig. 3), and then commanded the Omni robot to touch the bolt, simulating a tightening operation. After touching a bolt, the robot autonomously moved $80 \mathrm{~mm}$ up, advanced to the next waypoint (a position above the next bolt position in the pattern), and waited for the next command.

For each trial, participants were informed (by text on a computer screen) to do one of the following (randomly selected):

normal Place all four bolts in the scripted clockwise order.

skip Omit one indicated bolt.

order Place bolts in a different, indicated order.

inspect After clockwise placement, command robot to touch ("reinspect") one indicated bolt.

\subsubsection{Task B: Complex Bolt Insertion}

To explore a range of task complexities, we designed a version of the bolt insertion task with more variability and more command options. This task differed from Task A in that the bolt pattern consisted of six bolts (Fig. 4), increasing the number of possible transitions, and the assembly order was randomly selected with each task iteration. Because there was no fixed bolt order, the robot did not automatically advance after tightening a bolt. This task simulates an unscripted assembly operation, as might be found in repair or error correction tasks and in small-run manufacturing or prototyping. 


\subsubsection{Task C: Cooperative Positioning}

This task was designed to evaluate direct-touch commands in a cooperative task with continuous human input. In industrial assembly operations, workers often use lift-assist or similar devices to bear the weight of a large part or assembly but still guide the part into its final position. Current industrial solutions to this problem, observed in our visits to assembly facilities, commonly involve either a passive lift-assist device that bears the vertical load of the part but provides no other assistance, or an active device that the worker commands with a button interface. This task is a simplified representation of the types of cooperative manipulation tasks studied in Lee (2010) and Wojtara et al. (2009).

Participants commanded the robot to move to a target (a clear plastic cup), switched the robot into impedance control mode, and guided the robot into the cup to touch the bottom. After touching the bottom, the robot autonomously rose up out of the cup and returned to position control mode for the next movement command. This task utilized the same target positions as Task B but used only four targets, randomly positioned on the hexagonal pattern (Figure 5). Targets (cups) were placed approximately $30 \mathrm{~mm}$ away from hexagon vertices, so that participants could not simply move the robot straight down into each cup.

This task features a mode switch, from autonomous robot motion under position control to a cooperative mode under impedance control. Similar mode-switching behaviors have been demonstrated in other robotic systems (e.g., Frigola et al., 2006; Geravand et al., 2013; Haddadin et al., 2008), but to our knowledge, the use of physical interaction to trigger a mode switch has not been evaluated in a task context or compared with other mode-switching commands.

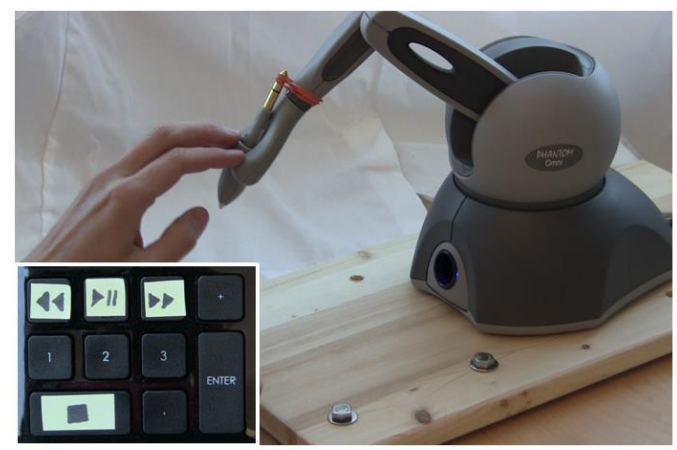

Figure 3. Experiment 1: Task A setup (simple bolt insertion).

Button interface is shown in inset.

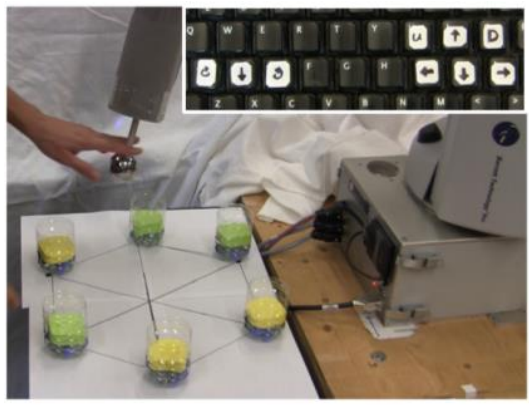

Figure 4. Experiment 2: Task B setup (complex bolt insertion).

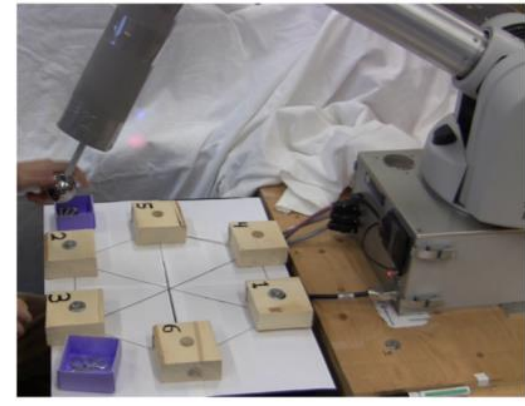

Figure 5. Experiment 2: Task C setup (collaborative positioning; button interface in insert). Four of six possible targets were used per trial. 


\subsection{Human-Robot Interaction Design}

We designed button and direct physical command interfaces for the three experimental tasks, as described below. See the supplementary video for demonstrations of the direct physical command schemes.

\subsubsection{Simple Bolt Insertion Task - Discrete Commands}

To conduct a fair comparison between physical and button commands, we designed the task and interface to feature a 1-to-1 mapping of tap and button commands. This limited physical commands (e.g., participants were not allowed to direct the robot diagonally across the workspace, because no such command was possible with the [binary] button controller).

Button Commands. The button interface employed three buttons for task execution (Fig. 3 inset) and a fourth button to deactivate the robot. Back/forward (clockwise motion) buttons moved the robot to the next/previous waypoint above a bolt. The play/pause button commanded the robot to touch ("tighten") a bolt or paused/resumed motion if the robot was moving. A stop button commanded the robot to return to the home position.

Direct Physical Commands. Users commanded the robot using only taps in various directions. Taps in the horizontal plane commanded the robot to move to the waypoint indicated by the direction of the tap, analogous to the left and right buttons. Users could only command the robot to move to orthogonally adjacent waypoints. A tap in the downward direction had the same function as the play/pause button. A tap in the upward direction had the same function as the stop button.

In pilot testing, we evaluated both directional taps and sustained pushes. Pushes were less usable due to the Omni's low stiffness and force saturation; we realized that more 'crisp' impedance was needed, and reserved this style for a later step on a stiffer robot.

\subsubsection{Complex Bolt Insertion Task - Discrete Commands}

Button Commands. Task A's scheme was reused, with two alterations: the stop button was unneeded, as users were no longer required to send the robot to the home position, and movement buttons were explicitly labeled as clockwise and counter-clockwise, as shown in Fig. 5 (inset). Users could command the robot to move to any arbitrary bolt by rapidly tapping the clockwise and counter-clockwise buttons (e.g., three quick taps would cause the robot to move directly to the opposite side of the bolt circle).

Direct Physical Commands. Experiment 1's scheme was reused, with one alteration: the robot was moved between waypoints using pushes (sustained contact, with robot displacement), rather than directional taps (brief contact, without robot displacement). In pilot testing, both interaction methods were effective on the WAM robot, but users were more successful in targeting waypoints using the push interaction. Only certain transitions were allowable, as was indicated by black lines around the target (Fig. 4). Bolt insertion orders were designed to be completed efficiently using only the allowable transitions.

\subsubsection{Cooperative Manipulation Task - Continuous Commands}

Button Commands. Participants moved the robot between waypoints similarly to Task B. Here, the down button switched the robot into fine control mode, at which point the robot executed a fast confirmatory "nod" (a dip of the end effector). In fine control mode, six buttons moved the robot in $3 \mathrm{~mm}$ increments in three dimensions (left, right, forward, back, up, and down). Step size was tuned in pilot testing to minimize task completion time. 
Direct Physical Commands. Participants moved the robot between waypoints as in Task B. A downward tap switched the robot into impedance mode, allowing the user to freely move the robot around the workspace; the mode switch was confirmed with a "nod," as for button commands.

\subsection{Experiment 1: Exploration of direct physical commands in simple collaboration}

To explore the specifics and user acceptance of a physical robot command scheme, participants executed Task A: Simple Bolt Insertion on the Omni robot, using direct physical command and button commands. Twenty volunteers ( 15 male, 5 female, mean age 27.9 years) were compensated $\$ 10.00$ for approximately 50-minute sessions. Participants had minimal to zero experience with robots (self-reported as $M=1.5, S D=1.2$ on a scale of 1 to 5 ) and none of them had ever encountered the particular model of robot used in our experiment.

\subsubsection{Experiment Design}

After initial training and familiarization with the robot and commands, participants completed one block of 10 trials for each command method (physical and button), with trials varying by task variant as noted above. Block order was counterbalanced among participants. When using button commands, half of participants wore the button controls on a belt, as would be the case in a "walk around" assembly task (although no walking was required in our task), and half had the buttons placed within easy reach next to the workspace, as for a smaller assembly task.

\subsubsection{Metrics}

Quantitative Metrics. We evaluated operational efficiency using task completion time, the total time from the start to the end of the task. As a measure of effective cooperation, we used cumulative concurrent assembly time. Concurrent motion, when both the human and the robot are in motion, is an effective measure of cooperation and interaction fluency (Hoffman \& Breazeal, 2007). In addition to this metric, we added the constraint that the human had to be both moving and executing an assembly operation (all robot motion counted as an assembly operation). This time was measured independently by two researchers viewing slow-motion video of the experiment. The measurements of the two researchers agreed well (Limit of Agreement $=0.14 \pm 0.07$ seconds, Pearsons's $r=0.93, p<0.001$ ). We used standard parametric methods (repeatedmeasures ANOVA and t-tests) to test the quantitative results. Reported effect sizes are simple differences in means.

Qualitative Metrics. After each block and at the experiments' end, participants answered questions about their impressions of the robot, collaborative quality, and method comparisons (Table 2). Parts of the questionnaire were adapted from Bartneck, Kulić, Croft, \& Zoghbi (2008) and Moon, Parker, Croft, \& Van der Loos (2013).

As recommended by Carifio and Perla (2007) and Norman (2010), we averaged responses to each group of questions to form three scores: Interaction (quality of interaction), Robot (participants' impression of the robot), and Preference (for command mode). We then used repeated-measures ANOVA and t-tests to test the results. In computing the Robot score, we reversed the scale on 'Dominant' and 'Forceful' so that 'good' responses mapped to higher numbers.

\subsection{Experiment 2: Complex Collaborative Tasks}

Our second experiment addressed the interplay between command mode and task parameters, evaluating physical commands in two different human-robot cooperative tasks. This experiment was designed based on results of our first experiment. Participants executed Task B: Complex Bolt Insertion and Task C: Cooperative Positioning on the WAM robot, using direct physical command and button commands. 
Gleeson et al. Haptic Control in H-R Collaborative Tasks

Table 2. Summary of questionnaire. Each question was answered on a 5-point Likert-type scale.

\begin{tabular}{|l|l|l|}
\hline $\begin{array}{l}\text { For each statement below, please For each word below, please Please answer the following } \\
\text { circle the number that indicates how indicate how well it describes } \\
\text { feel about your INTERACTION } \\
\text { with the robot. }\end{array}$ & $\begin{array}{l}\text { ROBOT you just worked with. } \\
\text { Eustions comparing the two-robot } \\
\text { control methods used in previous } \\
\text { task. }\end{array}$ \\
\hline It was easy to control the robot. & $\begin{array}{l}\text { Which method made it easier to } \\
\text { control the robot? }\end{array}$ \\
\hline $\begin{array}{l}\text { The robot understood what I wanted it } \\
\text { to do. }\end{array}$ & $\begin{array}{l}\text { Useful } \\
\text { to accomplish the task? }\end{array}$ \\
\hline $\begin{array}{l}\text { I always knew what the robot was } \\
\text { going to do next. }\end{array}$ & Dominant & $\begin{array}{l}\text { Which method made it easier for you } \\
\text { to concentrate on the task? }\end{array}$ \\
\hline I enjoyed working with the robot. & Forceful & $\begin{array}{l}\text { Which control method did you prefer } \\
\text { using overall? }\end{array}$ \\
\hline & Likeable & \\
\hline
\end{tabular}

Twenty-three volunteers (16 male, 7 female, mean age: 23.8 years), two of whom had participated in the first study, were compensated $\$ 10.00$ for approximately 50-minute sessions. Participants had minimal to zero experience with robots, (self-reported as $M=1.6, S D=1.1$ on a scale of 1 to 5) and none of them had ever encountered the particular model of robot used in our experiment.

\subsubsection{Experiment Design}

This experiment consisted of four conditions, testing all combinations of task (Task B or Task C) and command method (physical or button commands), where a trial comprised one task execution. After a training period, participants completed one five-trial block under each of the four conditions. We instructed participants to complete the task as quickly as possible. Blocks were grouped by task (e.g., a participant would complete both bolt blocks, one with each command method, before moving on to the positioning blocks), but the task and command mode orders were otherwise counterbalanced balanced between participants.

\subsubsection{Metrics}

Experiment 2 utilized the same quantitative and qualitative metrics as the first, with participants completing separate questionnaires for each of the two tasks. While the task completion time in the first experiment was delimited by the participant activating and deactivating the robot, participants in the second experiment explicitly timed themselves by starting and stopping a digital timer.

Here, we are most concerned with how an experienced user would perform. In our first experiment, we observed that task performance improved with experience, appearing to plateau after approximately four trials, so we measured performance based only the fifth iteration of each task (although the participants did not know this).

\section{Results and Discussion}

\subsection{Experiment 1: Exploration of direct physical commands in simple collaboration}

Our first experiment tested the practicality of direct physical commands for human-robot collaborative assembly tasks. Participants were able to successfully command the robot using touch. In general, button commands performed somewhat better, but there were few significant differences.

\subsubsection{Task Performance}

Task completion times were slightly better for button commands than for direct physical 
commands (Fig. 6). An ANOVA showed main effects of command method and task variation, as well as differences between participants and an interaction between command method and task variation (for all, $F>1.91, p<0.01$ ). Post hoc orthogonal paired t-tests show that button commands were reliably faster for the task without variation and for the 'inspection' variation (for both, $t>3.38, p<0.01$ ), although the effect size was small (1.82 and 3.61 seconds, respectively).

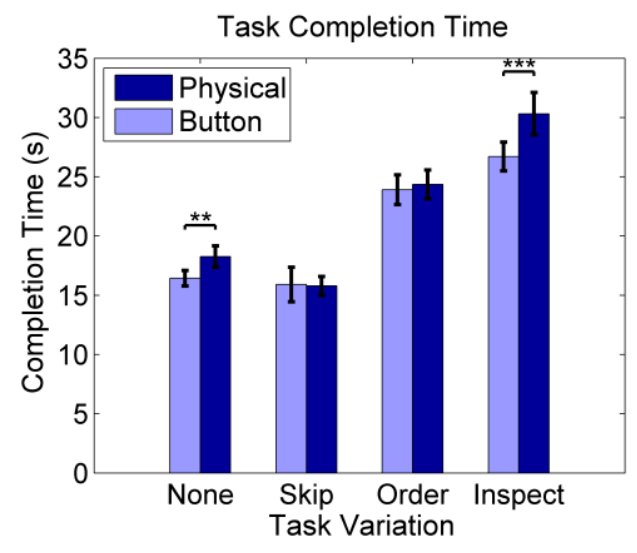

Figure 6. Experiment 1 (simple bolt insertion): Physical control performed slightly worse than button control, with faster completion times for button control in some task conditions.

Teamwork scores, computed as the proportion of the time that both human and robot were simultaneously working on the assembly task, are reported in Fig. 7. Again, there is some indication of better teamwork under button commands. An ANOVA showed main effects of all factors (participant, control mode, task variation), as well as interactions between all factors (for all, $F>2.02, p<0.05)$. In subsequent t-tests, command mode only had a reliable, but small, effect in tasks without variations, $46 \%$ vs. $41 \%,(t(88)=2.58, p=0.01)$.

This experiment also features a single between-subjects factor: the placement of the button controls either on a table next to the workspace or worn on a belt. Button placement did not affect task performance $(t(96)=0.61 p>0.50)$, with a mean effect of $0.30 \mathrm{~s}$ on task completion time.



Figure 7. Experiment 1 (simple bolt insertion): Button and physical control showed similar teamwork for task variations, but buttons did slightly better for non-varied task conditions. 


\subsubsection{User Preferences}

The results of user questionnaires are summarized in Fig. 8. On average, participants preferred button commands at marginal significance $(t(19)=1.94, p=0.07$, effect size $=0.6)$. Interaction and Robot scores were higher for button commands. Command method had a significant main effect on both scores, and both scores interacted significantly with participant (for all, $F>4.98, p<$ $0.05)$.
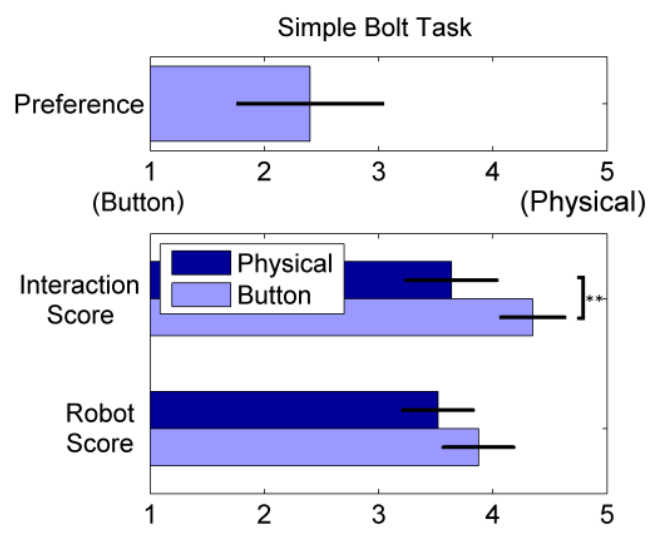

Figure 8. Experiment 1: Participants expressed a marginally significant preference for button control in the simple bolt insertion task.

\subsubsection{Discussion}

Potential benefit of physical commands. Our first study gave indications that users can successfully use physical interaction to command a robot in a collaborative task. Participants' comments confirmed our hypothesis that physical commands could improve engagement with the task and allow the users to better focus on the task, "[With physical commands, I] didn't have to divide attention between [the button] pad and [the] task ... I was working with the robot instead of controlling it" (P8).

Shortcomings in physical command implementation. The low stiffness and low precision of the Omni robot impeded effective physical command. Oscillation in the robot arm and noisy sensor readings resulted in command misidentification. Forty-five percent of participants commented on difficulties with the direct physical commands (e.g., "touch was not always clear to the robot. If it was, I think I would like touch better" [P6]). To resolve these difficulties, we conducted our second experiment on a robot with higher stiffness, greater force capabilities, and precision position sensors.

Interaction Method and Task Complexity. Based on our analysis, we hypothesize that the benefits of direct physical commands are only realized in tasks that require frequent, various, or continuous commands and where there is significant demand on the user's time and attention. Experiment 1's scripted bolt insertion was too simple to elicit these benefits. "Since the task was so easy, I couldn't find a preference [between command methods]" (P3). Additionally, because of the low robot speed, the human users had time to remove their hands and attention from the workspace to operate button controls. Users simply had no need for faster, more intuitive interaction commands. We conclude, therefore, that in simple, low-demand tasks that can be easily completed with buttons, button interfaces may be the preferred command method. Not all potential human-robot tasks are some simple and scripted, however, so in our second experiment, we study more complex, dynamic tasks. 
Robot Appearance. We chose the Omni robot because it is inherently safe. The harmless, plastic appearance of the robot seemed to make users comfortable with the robot: "The robot was very demure and non-threatening" (S19). However, some subjects expressed a fear of damaging the robot: "If you do not properly touch [the] robot ... might be [a] cause of damage to [the] robot" (S2). This prevented some participants from taping the robot with the required force. From this, we learn that the appearance of the robot is an important consideration for physical interaction; the robot should appear non-threatening but also robust.

Button Placement. We found no effect of button placement in our experiment and conclude that it is not an important factor in our tasks; either placement may be used with equal validity in subsequent experiments.

\subsection{Experiment 2: Complex Collaborative Tasks}

With a stiffer robot and less scripted tasks, physical commands performed better than button commands in both qualitative and quantitative measures. Specifically, physical commands provided a slight advantage in Task B (discrete bolt insertion) and a large advantage in Task C (continuous positioning).

\subsubsection{Task Performance}

Direct physical commands resulted in slightly faster completion times in Task B and much faster times in Task C (Fig. 9). An ANOVA found main effects of command mode, task and participant, with interactions between participant and task and between command mode and task (for all, $F$ $>2.31, p<0.05$ ). In post hoc paired t-tests, we found a small but significant effect of command mode in the complex bolt task $(t(44)=2.20, p<0.05$, effect size $=3.35 \mathrm{~s}, \sim 10 \%$ faster $)$ and a large effect in the positioning task $(t(44)=13.36, p<0.01$, effect size $=21.83 \mathrm{~s}, \sim 80 \%$ faster). Physical commands also improved teamwork (33\% vs. 20\%, Fig. 10). An ANOVA found main effects for both command mode and participant on the teamwork score, with a significant interaction (for all, $F>7.79, p<0.001$ ). Our teamwork metric was not relevant for the positioning task, which would always be zero for button commands.

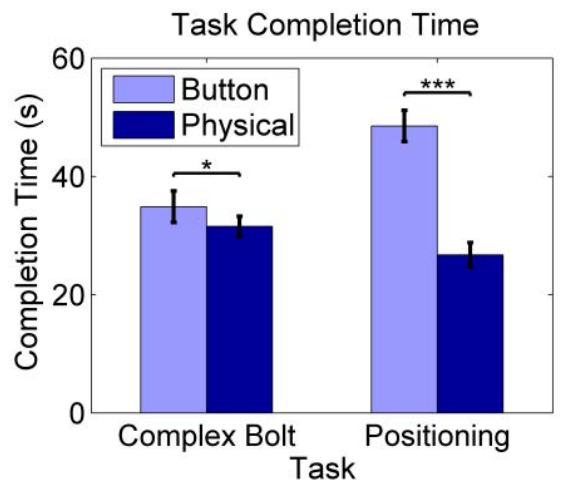

Figure 9. Experiment 2: Physical control was somewhat faster in the complex bolt insertion task and much faster for collaborative positioning.

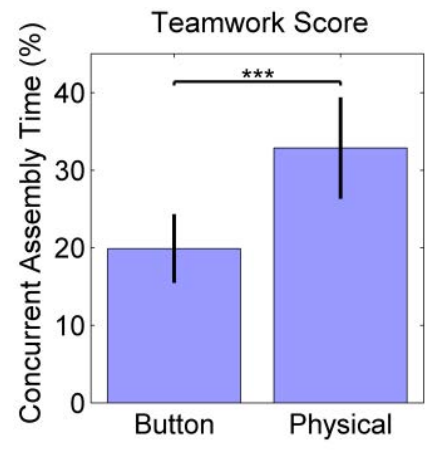

Figure 10. Experiment 2: Physical control significantly improved teamwork in the complex bolt task. 
Gleeson et al. Haptic Control in H-R Collaborative Tasks

\subsubsection{User Preferences}

User preferences were consistent with task performance (Fig. 11). The difference in preference scores between the two tasks was significant; an ANOVA showed main effects of task and participant, with interaction (for all, $F>2.39, p<0.05$ ). Subsequent t-tests showed physical commands preferred over buttons slightly for Task B (marginally significant at $\mathrm{t}(22)=1.71$, $\mathrm{p}=0.10$, effect size $=0.48)$ and heavily for Task $\mathrm{C}(t(22)=4.74, p<0.001$, effect size 1.07). An ANOVA showed no main effects on the Interaction score, and only the command mode affected Robot score $(F(1)=3.2, p<0.05)$. Considering the two tasks independently in subsequent t-tests, command mode had an effect on Interaction and Robot score in Task C (for both, $t(44)>2.16$, $p<0.05$ ) but not in Task B.

In Task B, preferences were bimodal, with $9 \%$ of participants strongly preferring button commands and 39\% physical control (composite Preference scores were $<1.5$, $>4.5$, respectively). In Task C, physical commands were the dominant favorite, with $57 \%$ of participants strongly preferring physical commands and zero participants strongly preferring button commands (Fig. 12).
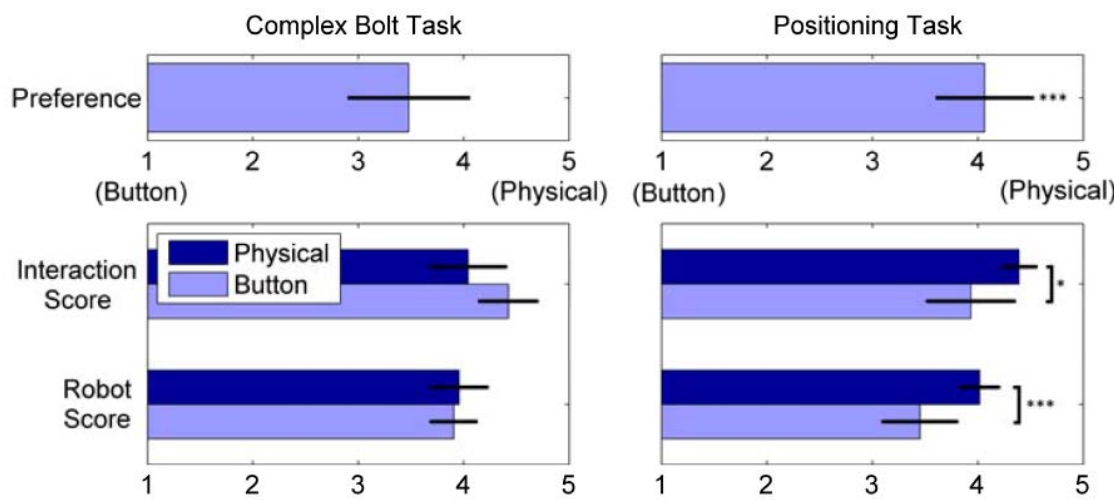

Figure 11. Experiment 2 qualitative results: Participants expressed a marginally significant preference for physical control in the complex bolt task and a large preference in the collaborative positioning task.

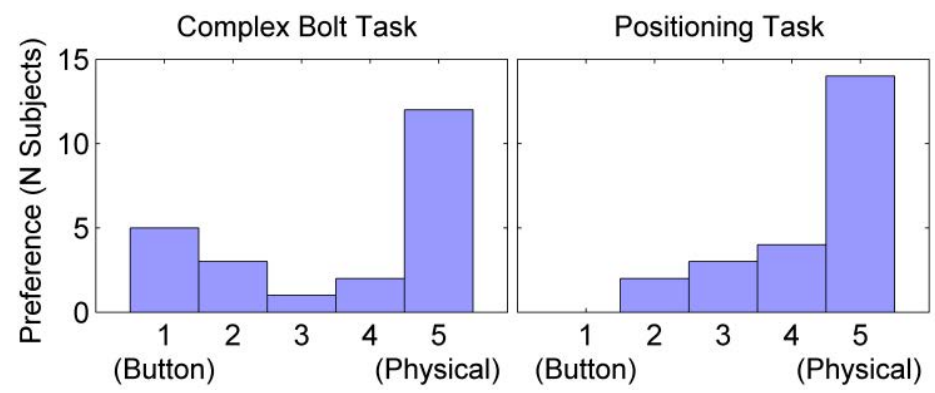

Figure 12. Experiment 2: Histograms for "Which control method did you prefer using overall?" Preferences are divided for bolt task and more uniform in the positioning task. These responses are representative of patterns for other survey questions.

\subsubsection{Discussion}

Benefit of physical commands. With a stiffer robot and tasks of greater complexity, physical commands improved task completion time and teamwork while further improving user experience. In contrast, button commands appears to have been more appropriate for the simpler, more scripted task of Experiment 1, together with the lower-stiffness robot. From this, we conclude that physical commands can be an important tool for human-robot collaborative work when tasks have inherent variation requiring significant human intervention. 
Participant comments support our conception of physical commands in robot collaboration as analogous to direct manipulation in $\mathrm{HCI}$, with benefits arising from the direct compatibility between user input and the desired robot response. "[Physical command] was more direct in the sense that I just needed to guide it and tap it instead of worrying about all of the buttons" (P9); "[There is] more direct manipulation with the touch control" (P16). However, button commands may provide a better sense of precision: "Touch control is faster, but button control is more precise" (S4).

The ability to directly manipulate the robot allowed users to more intuitively execute collaborative tasks and imposed lower cognitive demands. "Definitely more intuitive to push the robot around rather than controlling it with buttons" (P12); "[Physical command] was more intuitive” (P1, P22). Seventy-eight percent of participants reported that direct physical interaction made it easier to concentrate on the task. This allowed them to better focus their attention on the task and improved their ability to stay physically and visually engaged with the workspace, "[With physical command, I] don't have to divide attention between my hands and the target" (P14). Similarly, others expressed that the physical commands helped them to stay better engaged in the task, "I liked touch control, because I was working in the same space as [the] robot... With the button control I felt disconnected from [the] robot" (P17). For some users, the additional demands imposed by the button controls were a significant burden, "I was more worried about the [buttons] than the task itself" (P7).

The cognitive and engagement benefits of physical commands produced a quantitative teamwork improvement (Fig. 10) and improved impressions of the robot (Fig. 11); "With touch control, I was doing my own task and controlling the robot simultaneously" (P17); "[With physical commands] I got a chance to work with the robot, as opposed to telling [it] where to go next" (P17).

While there are performance benefits to physical commands, the familiarity of button commands is important for some users, "It is easier to control by buttons at the beginning, because I am used to a keyboard" (P7). In some tasks where user comfort is more important than performance, or when the task is simple, the familiarity of buttons may be preferable. Some users expressed a preference for button commands, even though they performed better with physical commands $(8,5)$; participants preferred button commands in the (bolt, positioning) task, even though some of them $(6,5)$ performed faster with physical commands. Two of these participants objected to the physical demands of the direct interaction, "buttons [require] less 'physical work"' (P16). For most users, however, the performance deficits of button commands were sufficient to outweigh the familiarity of the keyboard interface. "I really hated button control" (P23).

Interaction Method and Task Complexity. The benefit of physical commands appears to increase with overall task complexity. In Experiment 2, there is a strong interaction between command mode and task in both quantitative and qualitative results. Interpreting differences in results between our first and second experiment is impeded by the difference in robot, but the general trend is clear (Fig. 13).

It can be argued that buttons are a higher bandwidth interface than gesture or physical commands, so it is interesting that physical commands performed best in the tasks requiring the highest communication bandwidth. One interpretation of this trend is that direct physical commands remain easy and intuitive as task complexity increases, but button interfaces become more difficult to use when they become more complex.

Efficacy of physical interface implementation. The direct physical command method was much more effective when implemented on the WAM robot. While the contact detection method is not our focus, shortcomings potentially impacted the user experience as studied here. In Experiment 1, interaction failures were frequent, frustrating many users $(45 \%$ complained of physical command errors), and while the degree of impact on task performance is hard to assess, it was clearly adverse. Experiment 2 was less affected by sensing errors (23\% complained of physical command 
errors; for comparison, 27\% complained of confusion and other problems with the button interface). For our purposes, in addressing the potential utility of physical commands in general, it is encouraging that even an imperfect implementation can provide measurable benefits. We demonstrated that direct physical commands can be used on position-controlled robots without any additional sensors, but in applications where specialized sensors such as robotic skin are practical, we expect that the accuracy and utility of physical commands would improve significantly.

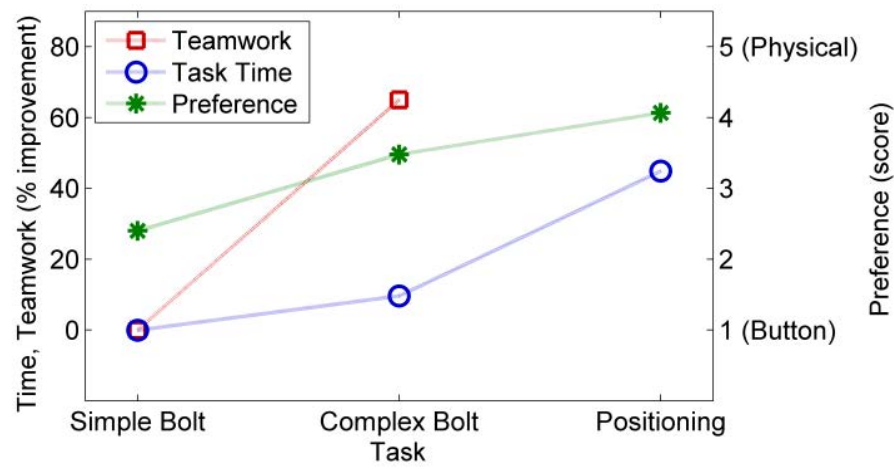

Figure 13. Summary of the increasing benefits of direct physical commands for more complex tasks, showing mean percent improvement in task completion times and teamwork scores (left axis) and mean preference scores (right axis). This figure presents a simplified depiction of task complexity; tasks varied on many dimensions (Table 1) and two different robots were employed.

Robot Appearance. Users interacted physically with a large, metal, high-speed robot arm. We anticipated that some participants would feel unsafe sharing a workspace with such a robot; however, no participants showed any signs of discomfort beyond some initial hesitation, and no one commented on feeling unsafe. How robot appearance may affect user perceptions is beyond our scope, but our results do provide anecdotal evidence that a user can feel comfortable when in close and physical contact with a larger, more powerful robot.

\section{Conclusions and Future Work}

Our results demonstrate a spectrum of appropriateness in command style, ranging from simple, low-option, scripted tasks where symbolic-type button commands work best to complex, interactive tasks where the human involvement extends beyond triggering advances, where direct physical commands can be an effective tool. It can improve task completion times, human-robot teamwork, and is often preferred by users. Physical commands are more intuitive than traditional button commands in more complex situations and allow users to better maintain physical and cognitive engagement with the task. We have accomplished the first step of demonstrating that the utility of physical robot commands clearly depends on the task; the next step is to establish, in greater detail, which task characteristics are most indicative of the preferred command method.

By demonstrating effective physical commands without direct force sensing or detailed robot models or controls, we show its feasibility for real-world applications, even on lower-cost position- controlled robots.

We have inferred, based on overall task performance and participant comments, that physical commands are more transparent and cognitively simpler than button commands. Future studies will confirm these assumptions by better characterizing the cognitive benefits of direct robot manipulation using haptic commands. While we designed experiments that broadly represent industrial applications, more work is required to understand how our results can be generalized to other tasks. 


\section{Acknowledgments}

This work has been possible thanks to support from General Motors and the Natural Sciences and Engineering Research Council of Canada under contract 140570247. We would like to thank Minhua Zheng for her help with the video analysis.

\section{References}

Adams, J. (2002). Critical considerations for human-robot interface development. In Proceedings of the AAAI Fall Symposium (pp. 1-8). North Falmouth, MA.

Azin, A., Balazs, D., Trygve, T., \& Gabor, S. (2012). New trends in industrial robot controller user interfaces. In Proceedings of the IEEE International Conference on Cognitive Infocommunications (CogInfoCom) (pp. 365-369). IEEE Press.

doi:10.1109/CogInfoCom.2012.6422007

Bartneck, C., Kulić, D., Croft, E., \& Zoghbi, S. (2008). Measurement instruments for the anthropomorphism, animacy, likeability, perceived intelligence, and perceived safety of robots. International Journal of Social Robotics, 1(1), 71-81. doi:10.1007/s12369-008- 0001-3

Bauer, A., Wollherr, D., \& Buss, M. (2008). Human-robot collaboration: A survey. International Journal of Humanoid Robotics, 5(1), 47-66. doi:10.1142/S0219843608001303

Bischoff, R., Kurth, J., Schreiber, G., Koeppe, R., Albu-Schaeffer, A., Beyer, A., . . Hirzinger, G. (2010). The KUKA-DLR Lightweight Robot Arm-A new reference platform for robotics research and manufacturing. In Proceedings of the International Symposium on Robotics (pp. 1-8). Munich, Germany.

Brogårdh, T. (2007). Present and future robot control development-An industrial perspective. Annual Reviews in Control, 31(1), 69-79. doi:10.1016/j.arcontrol.2007.01.002

Carifio, J., \& Perla, R. J. (2007). Ten common misunderstandings, misconceptions, persistent myths and urban legends about Likert scales and Likert response formats and their antidotes. Journal of Social Sciences, 3(3), 106-116. doi:10.3844/jssp.2007.106.116

Chan, W. P., Parker, C. A., Van der Loos, H. M., \& Croft, E. A. (2013). A human-inspired object handover controller. The International Journal of Robotics Research, 32(8), 971-983. doi:10.1177/0278364913488806

Chao, C., \& Thomaz, A. L. (2012). Timing in multimodal turn-taking interactions: Control and analysis using timed petri nets. Journal of Human-Robot Interaction, 1(1). doi:10.5898/jhri.v1i1.42

Chen, T. L., \& Kemp, C. C. (2010). Lead me by the hand: Evaluation of a direct physical interface for nursing assistant robots. In Proceedings of the IEEE International Conference on humanrobot interaction (pp. 367-374). IEEE Press. doi:10.1145/1734454.1734579

De Luca, A., Albu-Schaffer, A., Haddadin, S., \& Hirzinger, G. (2006). Collision detection and safe reaction with the DLR-III Lightweight Manipulator Arm. In Proceedings on the IEEE International Conference on Intelligent Robots and Systems (pp. 1623-1630). IEEE Press. doi:10.1109/IROS.2006.282053

Erden, M. S., \& Tomiyama, T. (2010). Human-intent detection and physically interactive control of a robot without force sensors. IEEE Transactions on Robotics, 26(2), 370-382. doi:10.1109/TRO.2010.2040202

Frémy, J., Ferland, F., Lauria, M., \& Michaud, F. (2014). Force-guidance of a compliant omnidirectional non-holonomic platform. Robotics and Autonomous Systems, 62(4), 579- 590. doi:10.1016/j.robot.2014.01.002 
Gleeson et al. Haptic Control in H-R Collaborative Tasks

Frigola, M., Casals, A., \& Amat, J. (2006). Human-robot interaction based on a sensitive bumper skin. In Proceedings of the IEEE/RSJ International Conference on Intelligent Robots and Systems (pp. 283-287). IEEE Press. doi:10.1109/IROS.2006.282139

Geravand, M., Flacco, F., \& de Luca, A. (2013). Human-robot physical interaction and collaboration using an industrial robot with a closed control architecture. In Proceedings of the IEEE International Conference on Robotics and Automation (ICRA) (pp. 3985-3992). doi:10.1109/ICRA.2013.6631141

Gleeson, B., MacLean, K., Haddadi, A., Croft, E., \& Alcazar, J. (2013). Gestures for industry: Intuitive human-robot communication from human observation. In Proceedings of the International Conference on Human-Robot Interaction (pp. 349-356). Tokyo, Japan.

Green, S. A., Billinghurst, M., Chen, X., \& Chase, G. J. (2008). Human-robot collaboration: A literature review and augmented reality approach in design. International Journal of Advanced Robotic Systems, 5, 1-18.

Haddadin, S., Albu-Schaffer, A., De Luca, A., \& Hirzinger, G. (2008). Collision detection and reaction: A contribution to safe physical human-robot interaction. In Proceedings of the IEEE International Conference on Intelligent Robots and Systems (pp. 3356-3363). IEEE Press. doi:10.1109/IROS.2008.4650764

Hoffman, G., \& Breazeal, C. (2007). Cost-based anticipatory action selection for human-robot fluency. IEEE Transactions on Robotics, 23(5), 952-961. doi:10.1109/TRO.2007.907483

Huber, M., Rickert, M., Knoll, A., Brandt, T., \& Glasauer, S. (2008). Human-robot interaction in handing-over tasks. In Proceedings of the 17th IEEE International Symposium on Robot and Human Interactive Communication (pp. 107-112). IEEE Press. doi:10.1109/ROMAN.2008.4600651

Hutchins, E., Hollan, J., \& Norman, D. (1985). Direct manipulation interfaces. Human-Computer Interaction, 1(4), 311-338. doi:10.1207/s15327051hci0104_2

Lee, S. (2010). MFR (Multi-purpose Field Robot) based on human-robot cooperative manipulation for handling building materials. Robot Manipulators, New Achievements (2010), 289-313.

Moon, A., Parker, C. A. C., Croft, E. A., \& Van der Loos, H. F. M. (2013). Design and impact of hesitation gestures during human-robot resource conflicts. Journal of Human-Robot Interaction, 2(3), 18-40. doi:10.5898/jhri.v2i3.49

Mukai, T., Onishi, M., Odashima, T., \& Hirano, S. (2008). Development of the tactile sensor system of a human-interactive robot "RI-MAN." IEEE Transactions on Robotics, 24(2), 505512. doi:10.1109/TRO.2008.917006

Nikolaidis, S., Lasota, P., Rossano, G., Martinez, C., Fuhlbrigge, T., \& Shah, J. (2013). Humanrobot collaboration in manufacturing: Quantitative evaluation of predictable, convergent joint action. In Proceedings of the 44th International Symposium on Robotics. Seoul, Korea. doi:10.1109/ISR.2013.6695625

Norman, G. (2010). Likert scales, levels of measurement and the "laws" of statistics. Advances in Health Sciences Education: Theory and Practice, 15(5), 625-32. doi:10.1007/s10459-0109222-y

Parker, C. A. C., \& Croft, E. A. (2012). Design \& personalization of a cooperative carrying robot controller. In Proceedings of the IEEE International Conference on Robotics and Automation (pp. 3916-3921). IEEE Press. doi:10.1109/ICRA.2012.6225120

Pieska, S., Kaarela, J., \& Saukko, O. (2012). Towards easier human-robot interaction to help inexperienced operators in SMEs. In Proceedings of the IEEE 3rd International Conference on Cognitive Infocommunications (CogInfoCom) (pp. 333-338). IEEE Press.

doi:10.1109/CogInfoCom.2012.6422002 
Gleeson et al. Haptic Control in H-R Collaborative Tasks

Shah, J., Wiken, J., Williams, B., \& Breazeal, C. (2011). Improved human-robot team performance using chaski, a human-inspired plan execution system. In Proceedings of the 6th International Conference on Human-Robot Interaction (p. 29). New York: ACM Press. doi:10.1145/1957656.1957668

Strabala, K., Lee, M. K., Dragan, A., Forlizzi, J., Srinivasa, S. S., \& Cakmak, M., \& Micelli, V. (2013). Toward seamless human-robot handovers, Journal of Human Robot Interaction 2(1), 112132. doi:10.5898/JHRI.2.1.Strabala

Suita, K., Yamada, Y., Tsuchida, N., Imai, K., Ikeda, H., \& Sugimoto, N. (1995). A failure-tosafety "Kyozon" system with simple contact detection and stop capabilities for safe humanautonomous robot coexistence. In Proceedings of the IEEE International Conference on Robotics and Automation (Vol. 3, pp. 3089-3096). IEEE Press. doi:10.1109/ROBOT.1995.525724

Voyles, R. M., \& Khosla, P. K. (1995). Tactile gestures for human/robot interaction. In Proceedings of the IEEE/RSJ International Conference on Intelligent Robots and Systems. Human Robot Interaction and Cooperative Robots (Vol. 3, pp. 7-13). IEEE Computer Society Press. doi:10.1109/IROS.1995.525854

Wojtara, T., Uchihara, M., Murayama, H., Shimoda, S., Sakai, S., Fujimoto, H., \& Kimura, H. (2009). Human-robot collaboration in precise positioning of a three-dimensional object.

Automatica, 45(2), 333-342. doi:http://dx.doi.org/10.1016/j.automatica.2008.08.021

Wrede, S., Emmerich, C., Grünberg, R., Nordmann, A., Swadzba, A., \& Steil, J. (2013). A user study on kinesthetic teaching of redundant robots in task and configuration space. Journal of Human-Robot Interaction, 2(1), 56-81. doi:10.5898/jhri.v2i1.98

Brian Gleeson ${ }^{1}$, brian.gleeson@gmail.com; Katelyn Currie ${ }^{2}$, kmcurrie.88@gmail.com; Karon MacLean ${ }^{1}$, maclean@cs.ubc.ca; Elizabeth Croft ${ }^{2}$, elizabeth.croft@ubc.ca; ${ }^{1}$ Department of Computer Science; ${ }^{2}$ Department of Mechanical Engineering, University of British Columbia, Vancouver, Canada. 